\title{
Sparse DOA estimation with polynomial rooting
}

\author{
Xenaki, Angeliki; Gerstoft, Peter; Fernandez Grande, Efren
}

\section{Published in:}

Proceedings of IEEE International Workshop on Compressed Sensing Theory and its Applications to Radar, Sonar and Remote Sensing

Link to article, DOI:

10.1109/CoSeRa.2015.7330273

Publication date:

2015

Document Version

Peer reviewed version

Link back to DTU Orbit

Citation (APA):

Xenaki, A., Gerstoft, P., \& Fernandez Grande, E. (2015). Sparse DOA estimation with polynomial rooting. In Proceedings of IEEE International Workshop on Compressed Sensing Theory and its Applications to Radar, Sonar and Remote Sensing [7330273] IEEE. https://doi.org/10.1109/CoSeRa.2015.7330273

\section{General rights}

Copyright and moral rights for the publications made accessible in the public portal are retained by the authors and/or other copyright owners and it is a condition of accessing publications that users recognise and abide by the legal requirements associated with these rights.

- Users may download and print one copy of any publication from the public portal for the purpose of private study or research.

- You may not further distribute the material or use it for any profit-making activity or commercial gain

- You may freely distribute the URL identifying the publication in the public portal 


\section{Sparse DOA estimation with polynomial rooting}

\author{
Angeliki Xenaki \\ Department of Applied Mathematics and \\ Computer Science \\ Technical University of Denmark \\ 2800 Kgs. Lyngby, Denmark \\ Email: anxe@dtu.dk
}

\author{
Peter Gerstoft \\ Scripps Institution of Oceanography \\ University of California San Diego \\ La Jolla, CA 92093-0238, USA
}

\author{
Efren Fernandez-Grande \\ Department of Electrical Engineering \\ Technical University of Denmark \\ 2800 Kgs. Lyngby, Denmark
}

\begin{abstract}
Direction-of-arrival (DOA) estimation involves the localization of a few sources from a limited number of observations on an array of sensors. Thus, DOA estimation can be formulated as a sparse signal reconstruction problem and solved efficiently with compressive sensing (CS) to achieve highresolution imaging. Utilizing the dual optimal variables of the CS optimization problem, it is shown with Monte Carlo simulations that the DOAs are accurately reconstructed through polynomial rooting (Root-CS). Polynomial rooting is known to improve the resolution in several other DOA estimation methods. However, traditional methods involve the estimation of the cross-spectral matrix hence they require many snapshots and stationary incoherent sources and are suitable only for uniform linear arrays (ULA). Root-CS does not have these limitations as demonstrated on experimental towed array data from ocean acoustic measurements.
\end{abstract}

\section{INTRODUCTION}

Sound source localization with sensor arrays involves the estimation of the direction-of-arrival (DOA) of (usually few) sources from a limited number of observations. Compressive sensing (CS) is a method for solving such underdetermined problems with a convex optimization procedure which promotes sparse solutions [1]. Solving the DOA estimation as a sparse signal reconstruction problem with CS, results in highresolution acoustic imaging [2]-[4], outperforming traditional methods [5] for DOA estimation.

To achieve computational efficiency along with very fine resolution, we solve the CS optimization problem in the dual domain [6]-[8]. We use the optimal dual variables to achieve high-resolution DOA estimation with the method of polynomial rooting. We refer to this method as Root-CS, similarly to established DOA estimation methods which are also based on polynomial rooting, namely Root-MVDR (minimum variance distortionless response beamformer), Root-MUSIC (multiple signal classification) and the minimum-norm method [5]. Even though these methods provide high resolution DOA estimation, they require the estimation of the cross-spectral matrix thus many snapshots and are suitable only for stationary incoherent sources. The estimation of the Root-CS solution is based on a single snapshot. Opposed to cross-spectral methods, single snapshot processing allows accurate reconstruction of moving sources and coherent arrivals. Moreover, Root-CS can be used with non-uniform arrays [8], [9] which is not possible for the established root methods.

Root-CS provides robust, high-resolution acoustic imaging even with a single snapshot as it is demonstrated both with
Monte Carlo simulations and with experimental data from ocean acoustic measurements.

\section{PROBLEM FORMULATION}

For simplicity, we assume plane wave propagation, i.e., the sources are in the far-field of the array, and narrowband processing with a known sound speed. Considering the onedimensional problem with a uniform linear array (ULA) of sensors, the location of a source is characterized by the DOA of the associated plane wave, $\theta \in\left[-90^{\circ}, 90^{\circ}\right]$, with respect to the array axis. The propagation delay from the $i$ th potential source to each of the $M$ array sensors is described by the steering vector

$$
\mathbf{a}\left(\theta_{i}\right)=\left[1, e^{j 2 \pi \frac{d}{\lambda} \sin \theta_{i}}, \cdots, e^{j 2 \pi \frac{d}{\lambda}(M-1) \sin \theta_{i}}\right]^{T},
$$

where $\lambda$ is the wavelength and $d$ is the intersensor spacing.

Discretizing the half-space of interest, $\theta \in\left[-90^{\circ}, 90^{\circ}\right]$, into $N$ angular directions the DOA estimation problem can be expressed with the linear model

$$
\mathbf{y}=\mathbf{A} \mathbf{x}+\mathbf{n}
$$

where $\mathbf{y} \in \mathbb{C}^{M}$ is the complex-valued data vector from the measurements at the $M$ sensors, $\mathbf{x} \in \mathbb{C}^{N}$ is the unknown vector of the complex source amplitudes at all $N$ potential DOAs and $\mathbf{n} \in \mathbb{C}^{M}$ is the additive noise vector. The sensing matrix

$$
\mathbf{A}_{M \times N}=\left[\mathbf{a}\left(\theta_{1}\right), \cdots, \mathbf{a}\left(\theta_{N}\right)\right],
$$

maps the complex source amplitudes $\mathbf{x}$ to the observations $\mathbf{y}$ and has as columns the steering vectors, Eq. (1), at all look directions. The noise is generated as independent and identically distributed (iid) complex Gaussian. The array signal-to-noise ratio (SNR) for a single-snapshot is used in the simulations, defined as $\mathrm{SNR}=20 \log _{10}\left(\|\mathbf{A} \mathbf{x}\|_{2} /\|\mathbf{n}\|_{2}\right)$.

\section{SPARSE DOA ESTIMATION}

The problem of DOA estimation is to recover the source vector $\mathbf{x} \in \mathbb{C}^{N}$ given the sensing matrix $\mathbf{A}$ and an observation vector $\mathbf{y} \in \mathbb{C}^{M}$. Practically, even though there are only a few sources $K<M$ generating the acoustic field, we are interested in a fine resolution on the angular grid to achieve accurate localization such that $M \ll N$ and the problem (2) is underdetermined. A way to solve this ill-posed inverse problem is to constrain the possible solutions with prior information. 
Traditional methods solve the underdetermined problem (2) by seeking the solution with the minimum $\ell_{2}$-norm which provides the best fit to the data,

$$
\min _{\mathbf{x} \in \mathbb{C}^{N}}\|\mathbf{y}-\mathbf{A} \mathbf{x}\|_{2}^{2}+\mu\|\mathbf{x}\|_{2}^{2}
$$

where $\mu \geq 0$ is a regularization parameter. The problem in Eq. (4) is convex with analytic solution $\widehat{\mathbf{x}}_{\ell_{2}}(\mu)=$ $\mathbf{A}^{H}\left(\mathbf{A} \mathbf{A}^{H}+\mu \mathbf{I}_{M}\right)^{-1} \mathbf{y}$, where $\mathbf{I}_{M}$ is the $M \times M$ identity matrix.

Conventional beamforming [5] (CBF) is the simplest source localization method. CBF combines the sensor outputs coherently to enhance the signal at a specific look direction from the ubiquitous noise and it is related to the $\ell_{2}$-norm method for large $\mu$,

$$
\widehat{\mathbf{x}}_{\mathrm{CBF}}=\lim _{\mu \rightarrow \infty}\left[\mu \widehat{\mathbf{x}}_{\ell_{2}}(\mu)\right]=\mathbf{A}^{H} \mathbf{y}
$$

$\mathrm{CBF}$ is robust to noise but suffers from low resolution and the presence of sidelobes.

Since $\mathbf{x}$ is sparse, it is appropriate to seek for the solution with the minimum $\ell_{0}$-norm, which counts the number of nonzero entries in the vector, to find a sparse solution. However, the $\ell_{0}$-norm minimization problem is a non-convex combinatorial problem which becomes computationally intractable even for moderate dimensions. The breakthrough of CS [1] came with the proof that for sufficiently sparse signals, $K<<N$, $K<M$, and sensing matrices with sufficiently incoherent (i.e., linearly independent) columns the $\ell_{0}$-norm minimization problem is equivalent (at least in the noiseless case) to its convex relaxation, the $\ell_{1}$-norm minimization problem, which can be solved efficiently with convex optimization even for large dimensions.

For noisy measurements, the $\ell_{1}$-norm minimization problem is formulated as [10]

$$
\widehat{\mathbf{x}}_{\ell_{1}}(\epsilon)=\underset{\mathbf{x} \in \mathbb{C}^{N}}{\arg \min }\|\mathbf{x}\|_{1} \text { subject to }\left\{\begin{array}{l}
\mathbf{y}=\mathbf{A x}+\mathbf{n}, \\
\|\mathbf{n}\|_{2} \leq \epsilon,
\end{array}\right.
$$

where $\epsilon$ is the noise floor. The estimate $\widehat{\mathbf{x}}_{\ell_{1}}(\epsilon)$ has the minimum $\ell_{1}$-norm while it fits the data up to the noise level.

CS offers high-resolution DOA estimation due to the sparsity constraint and computational efficiency due to convex relaxation of the $\ell_{0}$-norm optimization problem [2], [3], [11].

\section{ROOT-CS FOR DOA ESTIMATION}

Solving problem (6) in the dual domain can reduce the dimensionality of the problem significantly especially when fine angular resolution is required, $N \gg M$, thus improve the computational efficiency. To formulate the dual problem, first, we construct the Lagrangian by augmenting the objective function in Eq. (6) with a weighted sum of the constraint functions. The corresponding weights are the dual variables of the problem. Then, minimizing the Lagrangian over the primal optimization variable, $\mathbf{x}$, yields the dual function. The dual function gives a lower bound on the optimal value of problem (6) as it is the infimum of the Lagrangian. The dual problem finds the best lower bound by maximizing the dual function over the dual variables; see Ref. [8] for a detailed derivation of the dual problem.
The dual equivalent to problem (6) is [7], [8]

$$
\max _{\mathbf{c} \in \mathbb{C}^{M}} \operatorname{Re}\left(\mathbf{c}^{H} \mathbf{y}\right)-\epsilon\|\mathbf{c}\|_{2} \text { subject to }\left\|\mathbf{A}^{H} \mathbf{c}\right\|_{\infty} \leq 1 \text {. }
$$

By solving the dual problem (7), a vector $\widehat{\mathbf{c}} \in \mathbb{C}^{M}$ of dual variables is attained which is maximally aligned with the measurement vector $\mathbf{y} \in \mathbb{C}^{M}$ while its beamformed amplitude $\left|\mathbf{A}^{H} \widehat{\mathbf{c}}\right|$ is bounded by unity across the whole angular spectrum. Specifically, $\left|\mathbf{a}^{H}\left(\theta_{i}\right) \widehat{\mathbf{c}}\right|=1$ at the angular direction $\theta_{i}$ corresponding to the DOA of an existing source, $x_{i} \neq 0$ [8]. Thus, $\widehat{\mathbf{c}}$ is a a linear combination of the signal steering vectors, i.e., it resides in the signal subspace.

Note that $\mathbf{A}^{H} \mathbf{c}$ can be expressed as a trigonometric polynomial of the variable $z(\theta)=e^{-j 2 \pi \frac{d}{\lambda} \sin \theta}$, with coefficients the dual variables $\mathbf{c}=\left[c_{0}, \cdots, c_{M-1}\right]^{T}$ and degree $M-1$,

$$
H(z)=\mathbf{A}^{H} \mathbf{c}=\sum_{m=0}^{M-1} c_{m} z^{m}=\sum_{m=0}^{M-1} c_{m} e^{-j\left(2 \pi \frac{d}{\lambda} \sin \theta\right) m} .
$$

The inequality constraint in Eq. (7) implies that the dual polynomial has amplitude uniformly bounded for all $\theta \in$ $\left[-90^{\circ}, 90^{\circ}\right]$. Making use of results for bounded trigonometric polynomials [12], the constraint in Eq. (7) can be replaced with finite dimensional linear matrix inequalities,

$$
\begin{gathered}
\max _{\mathbf{c}, \mathbf{Q}} \operatorname{Re}\left(\mathbf{c}^{H} \mathbf{y}\right)-\epsilon\|\mathbf{c}\|_{2} \text { sub. to }\left[\begin{array}{cc}
\mathbf{Q}_{M \times M} & \mathbf{c}_{M \times 1} \\
\mathbf{c}_{1 \times M}^{H} & 1
\end{array}\right] \succeq 0, \\
\sum_{i=1}^{M-j} \mathbf{Q}_{i, i+j}= \begin{cases}1, & j=0 \\
0, & j=1, \cdots, M-1,\end{cases}
\end{gathered}
$$

which can be solved efficiently with semidefinite programming [13] to obtain an estimate for the coefficients, $\widehat{\mathbf{c}} \in \mathbb{C}^{M}$, of the dual polynomial. The number of optimization variables of the dual problem $(9)$ is $(M+1)^{2} / 2$ equal to half the number of elements of the matrix in the inequality constraint since this matrix is Hermitian.

\section{A. Support detection through the dual polynomial}

Strong duality assures that by solving the dual problem (7), or equivalently Eq. (9), we obtain the minimum of the primal problem (6). However, the dual problem provides an optimal dual vector, $\widehat{\mathbf{c}}$, but not the primal solution, $\widehat{\mathbf{x}}_{\ell_{1}}$. The corresponding dual polynomial has the properties [6]

$$
|H(z)| \leq 1, \forall \theta \in \mathbb{T} \rightarrow\left\{\begin{array}{l}
\left|H\left(z\left(\theta_{i}\right)\right)\right|=1, \theta_{i} \in \widehat{T} \\
|H(z(\theta))| \leq 1, \theta \in \mathbb{T} \backslash \widehat{T}
\end{array}\right.
$$

where $\mathbb{T}=\left[-90^{\circ}, 90^{\circ}\right]$ and $\widehat{T} \subset \mathbb{T}$ is the estimated set of DOAs. Thus, the support of the primal solution, i.e., the DOAs of the existing sources, is found by locating the angular directions where the amplitude of the dual polynomial (8) is one. This is achieved by locating the roots of the real valued nonnegative polynomial which lie on the unit circle [6], [12],

$$
P(z)=1-R(z)=1-\sum_{m=-(M-1)}^{M-1} r_{m} z^{m},
$$

where $R(z)=H(z) H(z)^{H}=|H(z)|^{2}$ with coefficients $r_{m}=\sum_{l=0}^{M-1-m} \widehat{c}_{l} \widehat{c}_{l+m}^{*}, m \geq 0$ and $r_{-m}=r_{m}^{*}$, i.e., the autocorrelation of $\widehat{\mathbf{c}}$. 

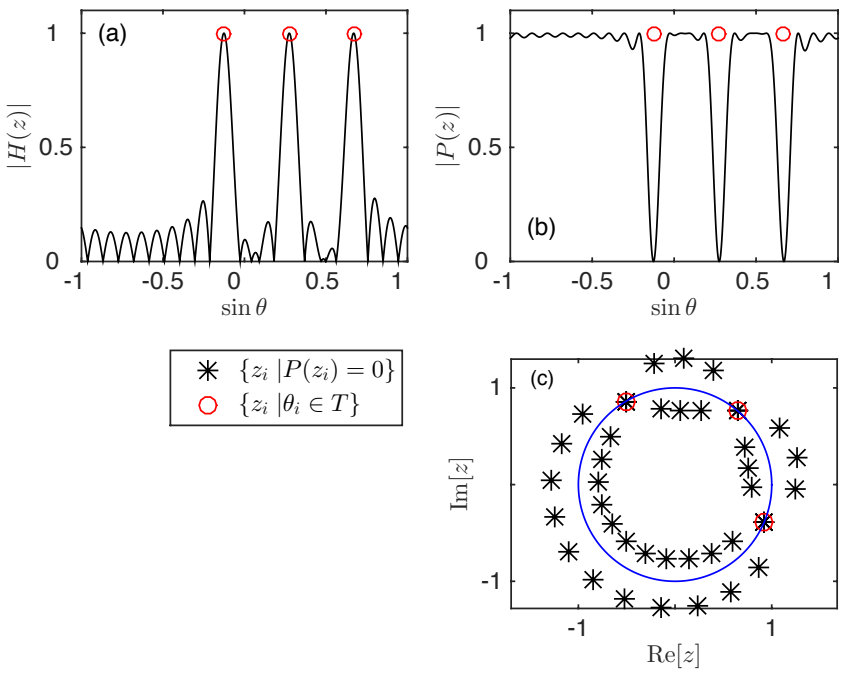

Fig. 1. Support detection through the dual polynomial. A standard ULA is used with $M=21$ sensors and $d / \lambda=1 / 2$ to localize three sources with unit strength and support set $T=\left[-7.24^{\circ}, 15.96^{\circ}, 42.07^{\circ}\right]$ and $\epsilon=0$. (a) The dual polynomial has amplitude one, $|H(z)|=1$, at $\theta_{i} \in T$. (b) Equivalently, $P(z)=0$ at $\theta_{i} \in T$. (c) The support $T$ is estimated by the angle of the roots, $z_{i}$, of $P(z)$ for which $\left|z_{i}\right|=1$

Note that the polynomial $P_{+}(z)=z^{M-1} P(z)$, of degree $2(M-1)$, which has only positive powers of the variable $z$, has the same roots as $P(z)$, besides the trivial root $z=0$. Therefore, the support of the solution, i.e., the DOAs of the existing sources, can be recovered by locating the roots of $P_{+}(z)$ on the unit circle (see Fig. 1),

$$
\widehat{T}=\left\{\theta_{i}=\sin ^{-1}\left(\frac{\lambda}{2 \pi d} \arg z_{i}\right)\left|P_{+}\left(z_{i}\right)=0,\right| z_{i} \mid=1\right\} .
$$

Figure 2 shows the robustness of support detection with Root-CS through Monte Carlo simulations for 1000 noise realizations for the configuration of Fig. 1. In the presence of noise, the solution is not unique and its support can include spurious DOAs, i.e., the support set has variable length depending on the noise realization. For high SNR the sources are always accurately detected (in this case Root-CS achieves accurate reconstruction even within a narrower bin width, i.e., $0.01^{\circ}$ ). For low SNR the DOAs are detected within a broader angular section around their true location (within 5 bins). This is due to the coherence (i.e., linear dependency) of neighboring steering vectors in the sensing matrix, Eq. (3), in combination with the high level of additive noise. Due to the variable length of the support set, some noisy artifacts along the angular spectrum appear with low frequency in both cases.

\section{B. Reconstruction of the primal solution}

Once the support is recovered (12) by locating the roots of the polynomial $P_{+}(z)$ which lie on the unit circle, the complex source amplitudes are recovered from

$$
\widehat{\mathbf{x}}_{\text {Root-CS }}=\mathbf{A}_{T}^{+} \mathbf{y}
$$

where ${ }^{+}$denotes the pseudoinverse of the matrix $\mathbf{A}_{T}$ with columns the steering vectors, Eq. (1), for $\theta_{i} \in \widehat{T}$.
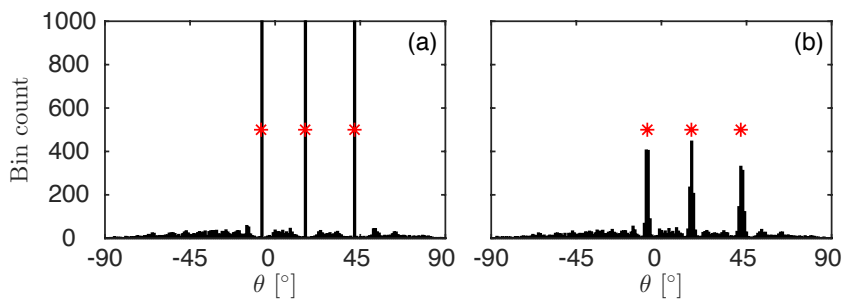

Fig. 2. Histograms for Root-CS DOA estimation based on 1000 Monte Carlo simulations for the configuration of Fig. 1 and bin width $1^{\circ}$; (a) $\mathrm{SNR}=20 \mathrm{~dB}$ $(\epsilon=0.83)$, (b) $\mathrm{SNR}=0 \mathrm{~dB}(\epsilon=8.3)$. The true DOAs are indicated $(*)$.

\section{Non-uniform arrays}

The method is also applicable to non-uniform arrays (constructed by randomly choosing sensors from a standard ULA configuration) by adding an additional constraint in the optimization problem (9). The additional constraint ensures that coefficients of the dual polynomial corresponding to inactive sensors on the ULA, $\mathbf{c}_{\text {null }}$, are annihilated [9],

$$
\begin{aligned}
& \max _{\mathbf{c}, \mathbf{Q}} \operatorname{Re}\left(\mathbf{c}^{H} \mathbf{y}\right)-\epsilon\|\mathbf{c}\|_{2} \text { sub. to }\left[\begin{array}{cc}
\mathbf{Q}_{M \times M} & \mathbf{c}_{M \times 1} \\
\mathbf{c}_{1 \times M}^{H} & 1
\end{array}\right] \succeq 0, \\
& \sum_{i=1}^{M-j} \mathbf{Q}_{i, i+j}=\left\{\begin{array}{ll}
1, & j=0 \\
0, & j=1, \cdots, M-1,
\end{array}, \mathbf{c}_{\text {null }}=0 .\right.
\end{aligned}
$$

\section{CROSS-SPECTRAL METHODS FOR DOA ESTIMATION}

Polynomial rooting can increase performance and achieve super-resolution in several DOA estimation methods, such as the minimum variance distortionless response (MVDR) beamformer, the multiple signal classification (MUSIC) method and the minimum-norm method. All these methods involve the estimation or the eigendecomposition of the cross-spectral matrix both in their spectral and root version.

The cross-spectral matrix estimated from $L$ snapshots (i.e., observations of $\mathbf{y}$ at a particular frequency) is defined as

$$
\widehat{\mathbf{C}}_{\mathbf{y}}=\frac{1}{L} \sum_{l=1}^{L} \mathbf{y}_{l} \mathbf{y}_{l}^{H} \text {. }
$$

The eigendecomposition of the cross-spectral matrix separates the signal and the noise subspaces,

$$
\widehat{\mathbf{C}}_{\mathbf{y}}=\widehat{\mathbf{U}}_{s} \widehat{\boldsymbol{\Lambda}}_{s} \widehat{\mathbf{U}}_{s}^{H}+\widehat{\mathbf{U}}_{n} \widehat{\boldsymbol{\Lambda}}_{n} \widehat{\mathbf{U}}_{n}^{H},
$$

where $\widehat{\mathbf{U}}_{s}$ comprises the signal eigenvectors, which correspond to the largest eigenvalues $\widehat{\boldsymbol{\Lambda}}_{s}$, and $\widehat{\mathbf{U}}_{n}$ comprises the noise eigenvectors. The signal eigenvectors are in the same subspace as the steering vectors, Eq. (1), while the noise eigenvectors are orthogonal to the subspace of the steering vectors, thus $\mathbf{a}(\theta)^{H} \widehat{\mathbf{U}}_{n}=\mathbf{0}$.

\section{A. Spectral version of DOA estimation methods}

MVDR [5] aims to minimize the output power of the beamformer under the constraint that the signal from the look direction remains undistorted. The MVDR beamformer power spectrum is

$$
P_{\text {MVDR }}(\theta)=\frac{1}{\mathbf{a}(\theta)^{H} \widehat{\mathbf{C}}_{\mathbf{y}}^{-1} \mathbf{a}(\theta)} .
$$


MUSIC [5] uses the orthogonality between the signal and the noise subspace to locate the maxima in the spectrum,

$$
P_{\text {MUSIC }}(\theta)=\frac{1}{\mathbf{a}(\theta)^{H} \widehat{\mathbf{U}}_{n} \widehat{\mathbf{U}}_{n}^{H} \mathbf{a}(\theta)} .
$$

The minimum-norm [5] is also an eigendecomposition based method but, unlike MUSIC which utilizes all noise eigenvectors, it uses a single vector, $\mathbf{v}=\left[v_{0}, \cdots, v_{M-1}\right]^{T}$, which resides in the noise subspace (compare with the dual vector $\widehat{\mathbf{c}}$ of Root-CS which resides in the signal subspace) such that

$$
\mathbf{a}\left(\theta_{i}\right)^{H} \mathbf{v}=0, \quad i=1, \cdots, K,
$$

where $K$ is the number of sources.

All the noise subspace eigenvectors, i.e., the columns of $\widehat{\mathbf{U}}_{n}$ have the property in Eq. (19). However, if the vector $\mathbf{v}$ is chosen as a linear combination of the noise subspace eigenvectors the algorithm tends to be more robust [14]. The minimumnorm method selects a vector, $\mathbf{v}$, in the noise subspace with minimum $\ell_{2}$-norm and unit first element, $v_{0}=1$. The vector $\mathbf{v}$ is constructed from the noise eigenvectors as

$$
\mathbf{v}=\widehat{\mathbf{U}}_{n} \mathbf{d}^{H} /\|\mathbf{d}\|_{2}^{2},
$$

where $\mathbf{d}$ is the first row of $\widehat{\mathbf{U}}_{n}$. The minimum-norm spectrum is

$$
P_{\text {min-norm }}(\theta)=\frac{1}{\mathbf{a}(\theta)^{H} \mathbf{v} \mathbf{v}^{H} \mathbf{a}(\theta)} .
$$

\section{B. Root version of DOA estimation methods}

The root version of the cross-spectral DOA estimation methods is based on the fact that for ULAs the null spectrum has the form of a nonnegative trigonometric polynomial. Thus, evaluating the spectrum is equivalent to evaluating the roots of the polynomial on the unit circle [15].

Analytically, let $N(\theta)=\mathbf{a}(\theta)^{H} \boldsymbol{\Psi} \mathbf{a}(\theta)$ be the null spectrum, such that the spectrum is $S(\theta)=N(\theta)^{-1}$. For MVDR, $\boldsymbol{\Psi}=\widehat{\mathbf{C}}_{\mathbf{y}}^{-1}$, for MUSIC, $\boldsymbol{\Psi}=\widehat{\mathbf{U}}_{n} \widehat{\mathbf{U}}_{n}^{H}$ and for the minimumnorm method, $\boldsymbol{\Psi}=\mathbf{v v}^{H}$ [5]. Then,

$$
\begin{aligned}
N(\theta) & =\sum_{m=0}^{M-1} \sum_{n=0}^{M-1} e^{-j 2 \pi m \frac{d}{\lambda} \sin \theta} \Psi_{m n} e^{-j 2 \pi n \frac{d}{\lambda} \sin \theta} \\
& =\sum_{l=-(M-1)}^{M-1} \psi_{l} e^{-j 2 \pi l \frac{d}{\lambda} \sin \theta} \\
N(z) & =\sum_{l=-(M-1)}^{M-1} \psi_{l} z^{l}
\end{aligned}
$$

where $\psi_{l}=\sum_{m-n=l} \Psi_{m n}$ is the sum of the elements of the Hermitian matrix $\boldsymbol{\Psi}$ along the $l$ th diagonal and $z(\theta)=$ $e^{-j 2 \pi(d / \lambda) \sin \theta}$.

The set of DOAs, $\widehat{T}$, is estimated from the roots of the polynomial $N(z)$, or equivalently the polynomial $N_{+}(z)=$ $z^{M-1} N(z)$, which lie on the unit circle, $z_{i}=e^{-j \arg \left(z_{i}\right)}$ as

$$
\widehat{T}=\left\{\theta_{i}=\sin ^{-1}\left(\frac{\lambda}{2 \pi d} \arg z_{i}\right)\left|N_{+}\left(z_{i}\right)=0,\right| z_{i} \mid=1\right\} .
$$

After the support is recovered, the amplitudes can be estimated through an overdetermined problem as in Eq. (13).

Even though the root forms of DOA estimation methods have, often, more robust performance than the corresponding spectral forms [16], they require a regular array geometry to form a trigonometric polynomial and detect its roots behavior. To achieve a robust estimate of the cross-spectral matrix many snapshots are required, $L>M$, i.e., stationary sources. Furthermore, eigendecomposition based methods fail to discern coherent arrivals. Forward/backward smoothing techniques [17] can be employed to mitigate this problem and make eigendecomposition based methods suitable for identification of coherent sources as well, but they still require a regular array geometry and an increased number of sensors.

\section{EXPERIMENTAL RESULTS}

The high-resolution capabilities of sparse signal reconstruction methods, i.e., CS for DOA estimation, even under noisy conditions and with random array configurations are demonstrated on ocean acoustic measurements. The interest is on single-snapshot reconstruction for source tracking and the results are compared with $\mathrm{CBF}$.

The data set is from the long range acoustic communications (LRAC) experiment [18] recorded from 10:00-10:30 UTC on 16 September 2010 in the NE Pacific. The data are from a horizontal uniform linear array towed at 3.5 knots at $200 \mathrm{~m}$ depth. The array has $M=64$ sensors, with intersensor spacing $d=3 \mathrm{~m}$. The data were acquired with a sampling frequency of $2000 \mathrm{~Hz}$ and the record is divided in $4 \mathrm{~s}$ nonoverlapping snapshots. Each snapshot is Fourier transformed with $2^{13}$ samples.

The data are post-processed with $\mathrm{CBF}$ (5), CS (6) and Root-CS (13) on a discrete DOA grid $\left[-90^{\circ}: 1^{\circ}: 90^{\circ}\right]$ at frequency $f=125 \mathrm{~Hz}(d / \lambda=1 / 4)$. The results are depicted in Fig. 3 both with all $M=64$ sensors active, Figs. 3(a)-(d) and by retaining only $M=16$ sensors active in a non-uniform configuration, Figs. 3(e)-(h). Both array configurations, Figs. 3(a) and 3(e), have the same aperture thus the same resolution.

The CBF map in Fig. 3(b) indicates the presence of three stationary sources at around $45^{\circ}, 30^{\circ}$ and $-65^{\circ}$. The two arrivals at $45^{\circ}$ and $30^{\circ}$ are attributed to distant transiting ships, while the broad arrival at $-65^{\circ}$ is from the towship. The CBF map suffers from low resolution and artifacts due to sidelobes and noise. The CS reconstruction ( $\epsilon=3.5$, Fig. 3(c)) results in improved resolution in the localization of the three sources by promoting sparsity and significant reduction of artifacts in the map. The Root-CS solution ( $\epsilon=3.5$, Fig. 3(d)) provides high resolution and further artifact reduction due to polynomial rooting.

Retaining only $1 / 4$ of the sensors on the array in a nonuniform configuration degrades the resolution of CBF due to increased sidelobe levels, Fig. 3(f). However, both CS on a discrete DOA grid, Fig.3(g), and Root-CS, Fig.3(h), provide high-resolution DOA estimation without a significant reconstruction degradation. The single-snapshot processing, Fig. 3, indicates that the sources are adequately stationary. Therefore, the 200 snapshots can be combined to estimate the cross-spectral matrix (15) and employ cross-spectral methods 


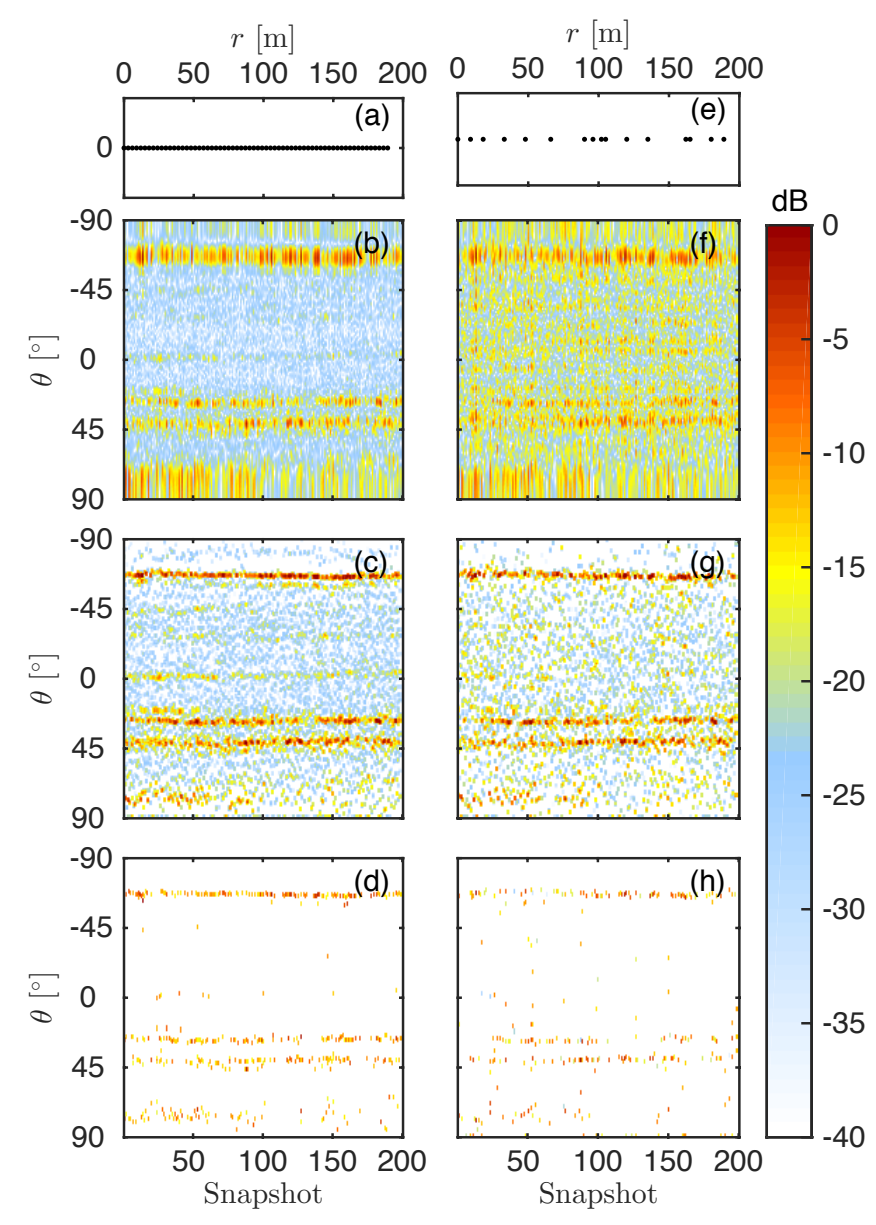

Fig. 3. Data from LRAC. (a) Uniform array with $M=64$ sensors and the corresponding (b) CBF, (c) CS on a discrete grid, $\left[-90^{\circ}: 1^{\circ}: 90^{\circ}\right]$, and (d) Root-CS reconstruction. (e) Non-uniform array with $M=16$ sensors and the corresponding (f) CBF, (g) CS and (h) Root-CS reconstruction.

for DOA estimation. Figure 4(a) compares the power spectra of MVDR (17), MUSIC (18) and the minimum-norm method (21) and Fig. 4(b) the corresponding root versions. The root versions of cross-spectral methods provide improved resolution compared to the corresponding spectral forms. However, the root cross-spectral methods require both many snapshots (i.e., stationary sources) for a robust estimate of the cross-spectral matrix and uniform arrays. Root-CS does not have these limitations.

\section{CONCLUSION}

DOA estimation with sensor arrays is a sparse signal reconstruction problem which can be solved efficiently with compressive sensing. Root-CS achieves high-resolution DOA estimation through the polynomial rooting method. In contrast to established DOA estimation methods, Root-CS provides high-resolution acoustic imaging even with non-uniform array configurations and robust performance under noisy measurements and single-snapshot data. This is demonstrated numerically with Monte Carlo simulations and validated with experimental data.

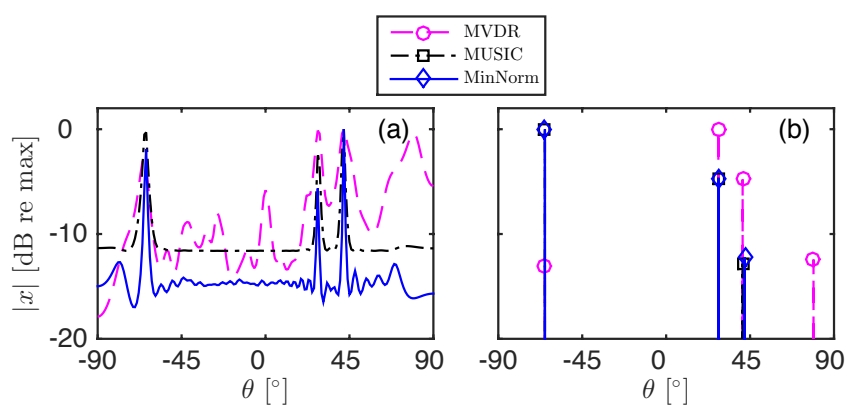

Fig. 4. Data from LRAC, combining the 200 snapshots to estimate the crossspectral matrix and processing with MVDR, MUSIC and the minimum-norm method. (a) Spectral version and (b) root version. The ULA with $M=64$ sensors and $d / \lambda=1 / 4$ is used.

\section{REFERENCES}

[1] M. Elad. Sparse and redundant representations: from theory to applications in signal and image processing, pages 1-359. Springer, New York, 2010.

[2] D. Malioutov, M. Çetin, and A. S. Willsky. A sparse signal reconstruction perspective for source localization with sensor arrays. IEEE Trans. Signal Process., 53(8):3010-3022, 2005.

[3] A. Xenaki, P. Gerstoft, and K. Mosegaard. Compressive beamforming. J. Acoust. Soc. Am., 136(1):260-271, 2014.

[4] C. F. Mecklenbräuker, P. Gerstoft, A. Panahi, and M. Viberg. Sequential Bayesian sparse signal reconstruction using array data. IEEE Trans. Signal Process., 61(24):6344-6354, 2013.

[5] H.L. Van Trees. Optimum Array Processing (Detection, Estimation, and Modulation Theory, Part IV), chapter 1-10. Wiley-Interscience, New York, 2002.

[6] E. J. Candès and C. Fernandez-Granda. Towards a mathematical theory of super-resolution. Comm. Pure Appl. Math., 67(6):906-956, 2014.

[7] E. J. Candès and C. Fernandez-Granda. Super-resolution from noisy data. J. Fourier Anal. Appl., 19(6):1229-1254, 2013.

[8] A. Xenaki and P. Gerstoft. Grid-free compressive beamforming. $J$. Acoust. Soc. Am., 137(4), 2015, DOI: 10.1121/1.4916269.

[9] G. Tang, B. N. Bhaskar, P. Shah, and B. Recht. Compressed sensing off the grid. IEEE Trans. Inf. Theory, 59(11):7465-7490, 2013.

[10] J. A. Tropp. Just relax: Convex programming methods for identifying sparse signals in noise. IEEE Trans. Inf. Theory, 52(3):1030-1051, 2006.

[11] S. Fortunati, R. Grasso, F. Gini, M. S. Greco, and K. LePage. Singlesnapshot DOA estimation by using compressed sensing. EURASIP J. Adv. Signal Process., 120(1):1-17, 2014.

[12] B. Dumitrescu. Positive trigonometric polynomials and signal processing applications, chapter 4.3. Springer, Dordrecht, Netherlands, 2007.

[13] S. Boyd and L. Vandenberghe. Convex optimization, pages 1-684. Cambridge university press, New York, 2004.

[14] S.S. Reddi. Multiple source location-a digital approach. IEEE Trans. Aerosp. Electron. Syst., 15(1):95-105, 1979.

[15] A. Barabell. Improving the resolution performance of eigenstructurebased direction-finding algorithms. In IEEE International Conference on Acoustics, Speech, and Signal Processing, ICASSP'83, volume 8, pages 336-339. IEEE, 1983.

[16] B. D. Rao and K. V. S. Hari. Performance analysis of root-MUSIC. IEEE Trans. Acoust., Speech, Signal Process., 37(12):1939-1949, 1989.

[17] B. D. Rao and K. V. S. Hari. Effect of spatial smoothing on the performance of MUSIC and the minimum-norm method. IEE Proc. Radar and Signal Proces., 137(6):449-458, 1990.

[18] H. C. Song, S. Cho, T. Kang, W. S. Hodgkiss, and J. R. Preston. Longrange acoustic communication in deep water using a towed array. $J$. Acoust. Soc. Am., 129(3):71-75, 2011. 